\title{
Fermi LAT Study of the Cosmic-rays and the Interstellar Medium in Nearby Molecular Clouds
}

\author{
K. Hayhashi ${ }^{a}$ and T. Mizuno on behalf of the Fermi-LAT collaboration \\ ${ }^{a}$ Department of Physical Science, Hiroshima University, Higashi-Hiroshima, Hiroshima 739-8526, Japan \\ ${ }^{b}$ Hiroshima Astrophysical Science Center, Hiroshima University, Higashi-Hiroshima, Hiroshima 739-8526, \\ Japan
}

\begin{abstract}
We report an analysis of the interstellar gamma-ray emission from nearby molecular clouds Chamaeleon, $\mathrm{R}$ Coronae Australis (R CrA), and Cepheus and Polaris flare regions with the Fermi Large Area Telescope (LAT). They are among the nearest molecular cloud complexes, within $\sim 300 \mathrm{pc}$ from the solar system. The gamma-ray emission produced by interactions of cosmic-rays (CRs) and interstellar gas in those molecular clouds is useful to study the CR densities and distributions of molecular gas close to the solar system. The obtained gamma-ray emissivities from $250 \mathrm{MeV}$ to $10 \mathrm{GeV}$ for the three regions are about $(6-10) \times 10^{-27}$ photons s $\mathrm{sr}^{-1} \mathrm{H}-$ atom $^{-1}$, indicating a variation of the CR density by $\sim 20 \%$ even if we consider the systematic uncertainties. The molecular mass calibration ratio, $X_{\mathrm{CO}}=N\left(\mathrm{H}_{2}\right) / W_{\mathrm{CO}}$, is found to be about $(0.6-1.0) \times 10^{20} \mathrm{H}_{2}$-molecule $\mathrm{cm}^{-2}\left(\mathrm{~K} \mathrm{~km} \mathrm{~s}^{-1}\right)^{-1}$ among the three regions, suggesting a variation of $X_{\mathrm{CO}}$ in the vicinity of the solar system. From the obtained values of $X_{\mathrm{CO}}$, we calculated masses of molecular gas traced by $W_{\mathrm{CO}}$ in these molecular clouds. In addition, similar amounts of dark gas at the interface between the atomic and molecular gas are inferred.
\end{abstract}

\section{INTRODUCTION}

Observation of high-energy gamma-rays from molecular clouds can be used to study the sources of cosmic rays (CRs), the CR density, and the distribution of the interstellar medium (ISM) in such systems. Gamma-rays are produced in the ISM by interactions of high-energy CR protons and electrons with the interstellar gas or the interstellar radiation field, via nucleon-nucleon collisions, electron bremsstrahlung, and inverse Compton (IC) scattering. Since the gamma-ray production cross section is almost independent of the thermodynamic state of the ISM, gamma-rays have been recognized as a powerful probe of the distribution of interstellar matter (e.g., Stecker 1970, Lebrun et al. 1983 and Blemen et al. 1984) If the gas column densities are estimated with good accuracy by observations in other wavebands such as radio and infrared, the $\mathrm{CR}$ spectrum and density distributions can be examined as well. Molecular clouds that are within $1 \mathrm{kpc}$ from the solar system (namely nearby molecular clouds) and have masses greater than a few $10^{3} M_{\odot}$ are well suited for an analysis of their gamma-ray emission to investigate the distribution of $\mathrm{CR}$ densities and interstellar gas since they are observed at high latitudes and therefore largely free from confusion with the strong emission from the Galactic plane. Study of such nearby molecular clouds in gamma-rays can be dated back to the COS-B era (Bloemen et al. 1984) and was advanced by the EGRET on board Compton Gamma-Ray Observatory (e.g., Hunter et al. 1994). Although some important information has been obtained on properties of CRs and the ISM by these early observations, detailed studies have only been performed on giant molecular clouds with masses greater than $\sim 10^{5} M_{\odot}$ such as the Orion complex (e.g., Digel et al. 1999).
The Large Area Telescope (LAT) (Atwood et al. 2009) on board the Fermi Gamma-ray Space Telescope has improved the situation significantly thanks to its unprecedented sensitivity. In addition, recent developments in studies of the ISM (e.g., Kalberla et al. 2005, Dame et al. 2001 and Grenier et al. 2005) allow us to investigate the CR spectra and density distribution with better accuracy.

Here, we report an analysis of Fermi LAT observations of the Chamaeleon, $\mathrm{R}$ Coronae Australis (R CrA), and Cepheus and Polaris flare molecular clouds. They are among the nearest $(\sim 300 \mathrm{pc}$ from the solar system) molecular clouds. No detailed study of $\mathrm{CR}$ and matter distributions for the Chamaeleon and $\mathrm{R}$ CrA regions had been performed before since they have rather small masses $\left(\lesssim 10^{4} M_{\odot}\right)$ and consequently small gamma-ray fluxes. We also analyzed in detail the region of the Cepheus and Polaris flares which was included in the Fermi LAT study of the second Galactic quadrant (Abdo et al. 2010a). Details of this proceedings are presented in a published paper (Ackermann et al. 2012a).

\section{DATA ANALYSIS}

For this analysis, we have accumulated events obtained from 2008 August 4 to 2010 May 9. During this time interval the LAT was operated in sky survey mode nearly all of the time and scanned the gammaray sky with relatively uniform exposure over time (within 10\% in regions studied). We used the stan- 
dard LAT analysis software, Science Tools ${ }^{1}$ version v9r16p0 and the response function P6_V3_DIFFUSE, which was developed to account for the detection inefficiencies due to pile-up and accidental coincidence of events (Rando et al. 2009). We set the lower energy limit at $250 \mathrm{MeV}$ to utilize good angular resolution (68\% containment radius is $\lesssim 1.5^{\circ}$ above 250 $\mathrm{MeV}$ ) and the upper energy limit at $10 \mathrm{GeV}$ because of limited photon statistics. The gamma-ray count maps obtained $(E>250 \mathrm{MeV})$ in the three regions are shown in Figure 1. Point sources with high significance (test statistic, $\mathrm{TS}^{2}$, greater than 50 ) are also plotted.

We modeled the gamma-ray emission as a linear combination of maps tracing the column density of the interstellar gas. This approach has been successfully applied in recent studies of diffuse gamma-rays by the LAT (e.g., Abdo et al. 2010a and Ackermann et al. 2011). With the usual assumptions of optical thinness and that CRs uniformly thread the ISM, gamma-ray intensity $\left(\mathrm{s}^{-1} \mathrm{~cm}^{-2} \mathrm{sr}^{-1} \mathrm{MeV}^{-1}\right)$ at a given energy can be modeled as

$$
\begin{aligned}
I_{\gamma}(l, b) & =\sum_{i=1}^{2} q_{\mathrm{HI}, i} \cdot N\left(\mathrm{H}_{\mathrm{I}}\right)(l, b)_{i}+q_{\mathrm{CO}} \cdot W_{\mathrm{CO}}(l, b) \\
& +q_{\mathrm{Av}} \cdot A \mathrm{v}_{\mathrm{res}}(l, b)+\mathrm{c}_{\mathrm{IC}} \cdot I_{\mathrm{IC}}(l, b) \\
& +I_{\mathrm{iso}}+\sum_{j} \mathrm{PS}_{j}
\end{aligned}
$$

where $i$ labels the regions along the line of sight separated in the analysis ( 1 for the Gould Belt and 2 for the rest). $q_{\mathrm{HI}, i}\left(\mathrm{~s}^{-1} \mathrm{sr}^{-1} \mathrm{MeV}^{-1}\right)$ and $q_{\mathrm{CO}}\left(\mathrm{s}^{-1} \mathrm{~cm}^{-2} \mathrm{sr}^{-1}\right.$ $\left.\mathrm{MeV}^{-1}\left(\mathrm{~K} \mathrm{~km} \mathrm{~s}^{-1}\right)^{-1}\right)$ are the emissivity per $\mathrm{H}$ I atom (traced by the $21 \mathrm{~cm}$ line of atomic hydrogen) and per $W_{\mathrm{CO}}$ unit (as a tracer of molecular gas), respectively. $q_{\text {Avres }}\left(\mathrm{s}^{-1} \mathrm{~cm}^{-2} \mathrm{sr}^{-1} \mathrm{MeV}^{-1} \mathrm{mag}^{-1}\right)$ is the emissivity per $A v_{\text {res }}$ magnitude which accounts for the gas not traced well by $\mathrm{H}$ i and $\mathrm{CO}$ : we constructed visual extinction $(A \mathrm{v})$ maps providing an estimate of the total column densities on the assumption of a constant gas-to-dust ratio, and obtained map by the fitting a linear combination of the $N(\mathrm{HI})$ and $W_{\mathrm{CO}}$ maps. $I_{\mathrm{IC}}$ and $I_{\text {iso }}$ are the IC model and isotropic background intensities $\left(\mathrm{s}^{-1} \mathrm{~cm}^{-2} \mathrm{sr}^{-1} \mathrm{MeV}^{-1}\right)$, respectively. We used GALPROP ${ }^{3}$ (e.g., Strong and Moskalenko 1998) for the IC model, and a publicly available isotropic

\footnotetext{
${ }^{1}$ Available from the Fermi Science Support Center (http://fermi.gsfc.nasa.gov/ssc/).

${ }^{2} \mathrm{TS}$ is defined as TS $=2\left(\ln L-\ln L_{0}\right)$, where $L$ and $L_{0}$ are the maximum likelihoods obtained with and without the source included in the model fitting, respectively; see (Mattox et al. 1996)

${ }^{3}$ http://www.mpe.mpg.de/ aws/propagate.html
}

spectrum $^{4}$ for the isotropic component. $\mathrm{PS}_{j}$ represents contributions of point sources included in the 1FGL catalog (Abdo et al. 2010b). See Ackermann et al. (2012a) for details of the analysis.

\section{RESULTS}

Figure 2(left) shows the obtained gamma-ray emissivity spectra per $\mathrm{H}$-atom of each region assuming the spin temperature $T_{\mathrm{s}}=125 \mathrm{~K}$. The emissivities above $250 \mathrm{MeV}$ are $\left(5.9 \pm 0.1_{\text {stat }}{ }_{-1.0 \text { sys }}^{+0.9}\right) \times 10^{-27}$ photons s ${ }^{-1} \mathrm{sr}^{-1} \mathrm{H}_{\text {-atom }}{ }^{-1},\left(10.2 \pm 0.4_{\text {stat }}{ }_{-1.7}^{+1.2}\right.$ sys $) \times$ $10^{-27}$ photons s $^{-1} \mathrm{sr}^{-1} \mathrm{H}$-atom ${ }^{-1}$, and $\left(9.1 \pm 0.3_{\text {stat }}\right.$ $\left.{ }_{-0.6 \text { sys }}^{+1.5}\right) \times 10^{-27}$ photons s${ }^{-1} \mathrm{sr}^{-1} \mathrm{H}$-atom ${ }^{-1}$ for the Chamaeleon, R CrA, and Cepheus and Polaris flare regions, respectively. In order to examine the systematic uncertainty due to the optical depth correction, we also tried to fit the data with maps obtained by assuming $T_{\mathrm{s}}=100 \mathrm{~K}$ and under the approximation that the gas is optically-thin. We evaluated the uncertainty of the isotropic component to be $\pm 10 \%$ by comparing the model we adopted and those derived in other LAT studies of mid-latitude regions (Abdo et al. 2009a and Abdo et al. 2009b). We thus reran the analysis assuming a $10 \%$ higher and lower intensity for the fixed isotropic component. We also investigated the effect on the systematic uncertainty due to the IC component by using different IC model maps which are constructed under different assumptions about the distribution of CR sources and intensity of the interstellar radiation field (e.g., Ackermann et al. 2012b). The effects of the uncertainty of the $T_{\mathrm{s}}$, isotropic component, and IC models are quite comparable, therefore we added them. The resultant peak-to-peak uncertainty of the local $\mathrm{H}_{\mathrm{I}}$ emissivity is less than $\sim 20 \%$ across the energy range for three regions investigated.

\section{DISCUSSION}

\subsection{CR Density and Spectrum Close to the Solar System}

We then compared the obtained $\mathrm{H}$ I emissivity spectra among the three regions, and model spectra ${ }^{5}$ for the local interstellar spectrum (LIS) as shown in Figure2 (left). Whereas the spectral shapes for the three

\footnotetext{
${ }^{4}$ isotropic_iem_v02.txt from http://fermi.gsfc.nasa.gov/ssc/ data/access/lat/BackgroundModels.html

${ }^{5}$ The model is calculated from the LIS compatible with the CR proton spectrum measured by Alcaraz et al. (2000) and Sanuki et al. (2000), under the assumption that the nuclear enhancement factors are 1.45 and 1.84 (Mori 2009); see Abdo et al. (2009b).
} 

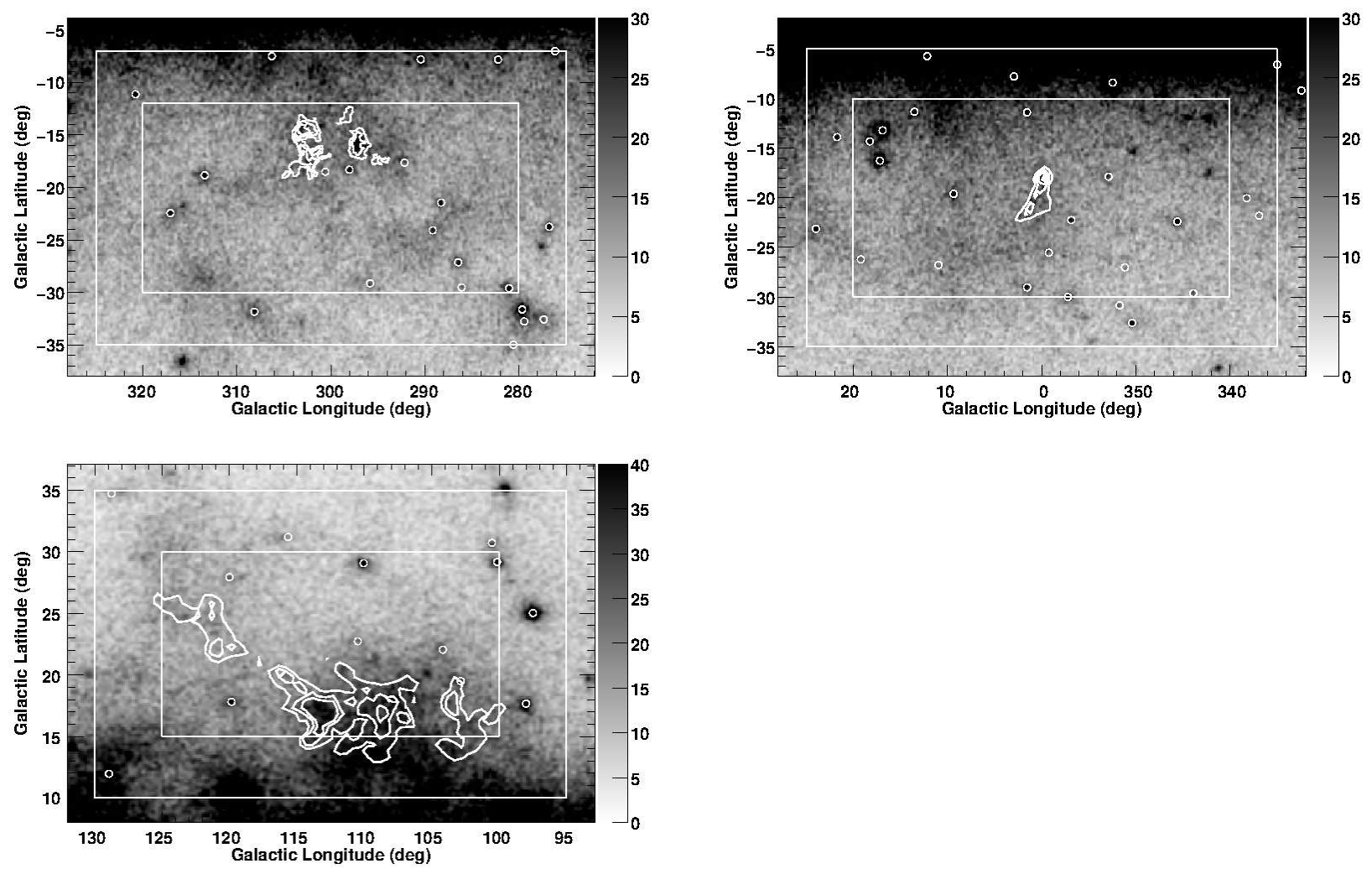

Figure 1: Gamma-ray count maps above $250 \mathrm{MeV}$ for the Chamaeleon (top left), R CrA (top right), and Cepheus and Polaris flare (bottom left) regions, smoothed with a Gaussian of $\sigma=0.5^{\circ}$ for display. The contours indicate intensities $W_{\mathrm{CO}}$ of the $2.6 \mathrm{~mm}$ line of $\mathrm{CO}$ (with the levels of $4,8,12$, and $16 \mathrm{~K} \mathrm{~km} \mathrm{~s}^{-1}$ ) by Dame et al. (2001), as a standard tracer of the molecular gas. White circles show the positions of point sources with high significance (TS $\geq 50)$ in the First Fermi LAT catalog (1FGL) by Abdo et al. (2010b). The inner squares indicate the ROI analyzed for each of the regions. Point sources outside of this ROI but inside the outer square are taken into account in the analysis.

regions studied here agree well with the LIS model, the absolute emissivities differ among the three regions. We note that the systematic uncertainty of the LAT effective area (5\% at $100 \mathrm{MeV}$ and $20 \%$ at 10 GeV; (Rando et al. 2009) does not affect the relative value of emissivities among these regions. The effect of unresolved point sources is small, since we have verified that the obtained emissivities are robust against the lower threshold for point sources between $\mathrm{TS}=50$ and 100. Although the emissivities of the $\mathrm{R}$ CrA region and the Cepheus and Polaris flare region are comparable, that of the Chamaeleon region is lower by $\sim 20 \%$, even if we take the systematic uncertainties into account. Therefore, the LAT data point to variations in the $\mathrm{CR}$ densities within $\sim 300$ $\mathrm{pc}$ in the Gould Belt. If the CR density has a variation by a factor of $\sim 1.2$ in the neighborhood of the solar system, this requires a serious reconsideration of a smooth CR density often adopted for simplicity, and may have an impact on the study of the CR source distribution and diffuse gamma-ray emission. We note that CR sources are stochastically distributed in space and time, and this may produce a CR anisotropy de- pending on the propagation conditions (e.g., Blasi \& Amado 2012a and Blasi \& Amato 2012b). Study of other regions and more detailed theoretical calculations are needed for further investigating this issue. On the other hand, we need to further investigate possible systematic uncertainties due to variations in $X_{\mathrm{CO}}$ (see the next paragraph) or dust-to-gas ratio we assumed in the construction of the $A \mathrm{v}_{\text {res }}$ map.

\subsection{Molecular Masses in the Interstellar Clouds Studied}

Under the hypothesis that the same CR flux penetrates the $\mathrm{HI}$ and $\mathrm{CO}$ phases of an interstellar complex, we can calculate the molecular mass calibration ratio, $X_{\mathrm{CO}}$, as $X_{\mathrm{CO}}=q_{\mathrm{CO}} /\left(2 q_{\mathrm{HI}}\right)$. As shown in Figure 2 (right), we derived the $X_{\mathrm{CO}}$ values from a linear fit of the $\left(q_{\mathrm{HI}}, q_{\mathrm{CO}}\right)$ points. The obtained $X_{\mathrm{CO}}$ values are summarized in Table I) $\left(0.96 \pm 0.06_{\text {stat }}\right.$ $\left.{ }_{-0.12 \mathrm{sys}}^{+0.15}\right) \times 10^{20} \mathrm{~cm}^{-2}\left(\mathrm{~K} \mathrm{~km} \mathrm{~s}^{-1}\right)^{-1},\left(0.99 \pm 0.08_{\text {stat }}\right.$ $\left.{ }_{-0.10 \mathrm{sys}}^{+0.18}\right) \times 10^{20} \mathrm{~cm}^{-2}\left(\mathrm{~K} \mathrm{~km} \mathrm{~s}^{-1}\right)^{-1}$, and $(0.63 \pm$ $\left.0.02_{\text {stat }}{ }_{-0.07 \mathrm{sys}}^{+0.09}\right) \times 10^{20} \mathrm{~cm}^{-2}\left(\mathrm{~K} \mathrm{~km} \mathrm{~s}^{-1}\right)^{-1}$ for the 

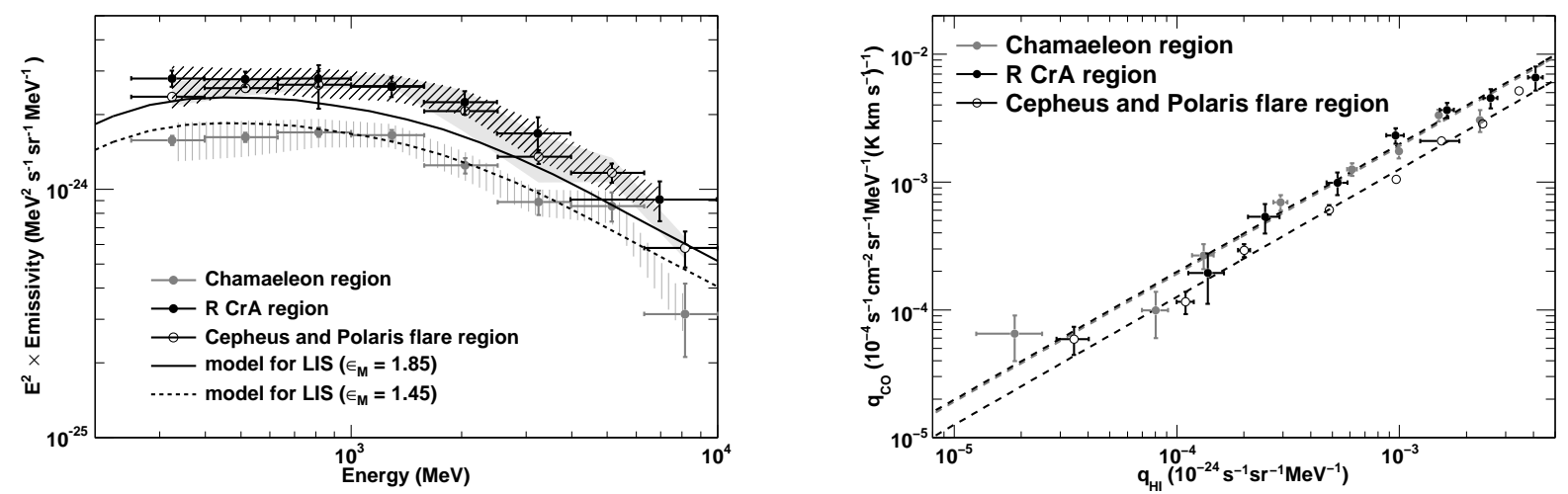

Figure 2: (left): H I emissivity spectra of each region compared with the model for LIS with the nuclear enhancement factors of 1.45 and 1.84 (Mori 2009). The shaded areas indicate the systematic uncertainty: vertical lines (Chamaeleon), diagonal lines (R CrA) and the gray area (Cepheus and Polaris flare). (right): CO versus H I emissivities. Each point corresponds to an energy bin. Errors are statistical only.

Chamaeleon, R CrA, and Cepheus and Polaris flare regions, respectively. The obtained value of $X_{\mathrm{CO}}$ for the Cepheus and Polaris flare region is $\sim 20 \%$ lower than that reported by Abdo et al. (2010a). Abdo et al. (2010a) includes in their study also the Cassiopeia molecular cloud in the Gould Belt, and due to different ROIs considered, the $q_{\mathrm{HI}}$ emissivity was also different. $X_{\mathrm{CO}}$ of the Chamaeleon region is similar to that of the $\mathrm{R}$ CrA region, whereas that of the Cepheus and Polaris flare region is $\sim 2 / 3$ of the others. The LAT data thus suggest a variation of $X_{\mathrm{CO}}$ on a $\sim 300 \mathrm{pc}$ scale.

Using these $X_{\mathrm{CO}}$ values, the molecular masses traced by $W_{\mathrm{CO}}$ can be calculated. The mass of the gas traced by $W_{\mathrm{CO}}$ is expressed as

$$
\frac{M}{M_{\odot}}=2 \mu \frac{m_{\mathrm{H}}}{M_{\odot}} d^{2} X_{\mathrm{CO}} \int W_{\mathrm{CO}}(l, b) d \Omega
$$

where $d$ is the distance to the cloud, $m_{\mathrm{H}}$ is the mass of the hydrogen atom and $\mu=1.36$ is the mean atomic weight per H-atom (Allen 1973). From this equation the mass of gas traced by $\mathrm{CO}$ is expressed as $M_{\mathrm{CO}}$ in Table If we obtained $\sim 5 \times 10^{3} M_{\odot}, \sim$ $10^{3} M_{\odot}$, and $\sim 3.3 \times 10^{4} M_{\odot}$ for the Chamaeleon, $\mathrm{R}$ CrA, and Cepheus and Polaris flare regions, respectively. From the linear relation between $q_{\mathrm{HI}}$ and $q_{\text {Avres }}$ $\left(q_{\text {Avres }}=X_{\text {Avres }} \cdot q_{\mathrm{HI}}\right)$ we can calculate the masses of additional gas traced by $q_{\text {Avres }}$ with a procedure similar to that for $\mathrm{CO}$ and results are summarized in Table 1. We thus obtained mass estimates for the Chamaeleon and $\mathrm{R}$ CrA regions similar to previous ones (Dame et al. 1987) if we consider the total mass (traced by $W_{\mathrm{CO}}$ and $A \mathrm{v}_{\text {res }}$ ), valthough the procedure is not straightforward since the gas traced by $A v_{\text {res }}$ is extended in a much larger region of the sky. Detailed study of the matter distribution in the interstellar space by comparing gamma-rays and other tracers will be reported elsewhere.

\section{Summary and Conclusions}

We have studied the gamma-ray emission from the Chamaeleon, R CrA, and Cepheus and Polaris flare molecular clouds close to the solar system $(\lesssim 300$ pc $)$ using the first 21 months of Fermi LAT data. Thanks to the excellent performance of the LAT, we have obtained unprecedentedly high-quality emissivity spectra of the atomic and molecular gas in these regions in the $250 \mathrm{MeV}-10 \mathrm{GeV}$ range.

The gamma-ray emissivity spectral shapes in three regions agree well with the model for the LIS (a model based on local CR measurement), thus indicating a similar spectral distribution of CRs in these regions. The emissivities, however, indicate a variation of the CR density of $\sim 20 \%$ within $\sim 300$ pc around the solar system, even if we consider the systematic uncertainties. We consider possible origins of the variation are non-uniform supernova rate and anisotropy of CRs depending on the propagation conditions.

The molecular mass calibration ratio $X_{\mathrm{CO}}$ for the Chamaeleon cloud and the R CrA cloud are comparable, whereas that of the Cepheus and Polaris flare region is $\sim 2 / 3$ of the others, suggesting a variation of $X_{\mathrm{CO}}$ in the vicinity of the solar system. From the obtained values of $X_{\mathrm{CO}}$, the masses of gas traced by $W_{\mathrm{CO}}$ in the Chamaeleon, $\mathrm{R} \mathrm{CrA}$, and Cepheus and Polaris flare regions are estimated to be $\sim 5 \times 10^{3}$ $M_{\odot}, \sim 10^{3} M_{\odot}$, and $\sim 3.3 \times 10^{4} M_{\odot}$ respectively. Similar amounts of gas are inferred to be in the phase not well traced by the $\mathrm{H}$ i or $\mathrm{CO}$ lines. Accumulation of more gamma-ray data, particularly at high energies, and progress in ISM studies, will reveal the CR and matter distribution in greater detail. 
Table I $X_{\mathrm{CO}}, X_{\mathrm{Avres}}$ and masses in the interstellar clouds in each region

\begin{tabular}{ccccc}
\hline \hline & $\begin{array}{c}X_{\mathrm{CO}} \\
\left(\times 10^{20} \mathrm{~cm}^{-2}\left(\mathrm{~K} \mathrm{~km} \mathrm{~s}^{-1}\right)^{-1}\right)\end{array}$ & $\begin{array}{c}M_{\mathrm{CO}} \\
\left(M_{\odot}\right)\end{array}$ & $\begin{array}{c}X_{\text {Avres }} \\
\left(\times 10^{22} \mathrm{~cm}^{-2} \mathrm{mag}^{-1}\right)\end{array}$ & $\begin{array}{c}M_{\text {Avres }} \\
\left(M_{\odot}\right)\end{array}$ \\
\hline Chamaeleon & $0.96 \pm 0.06_{\text {stat }} \pm 0.13_{\text {sys }}$ & $\sim 5 \times 10^{3}$ & $0.22 \pm 0.01_{\text {stat }} \pm 0.08_{\text {sys }}$ & $\sim 2.0 \times 10^{4}$ \\
R CrA & $0.99 \pm 0.08_{\text {stat }} \pm 0.14_{\text {sys }}$ & $\sim 10^{3}$ & $0.21 \pm 0.01_{\text {stat }} \pm 0.02_{\text {sys }}$ & $\sim 10^{3}$ \\
Cepheus \& Polaris & $0.63 \pm 0.02_{\text {stat }} \pm 0.08_{\text {sys }}$ & $\sim 3.3 \times 10^{4}$ & $0.14 \pm 0.01_{\text {stat }} \pm 0.03_{\text {sys }}$ & $\sim 1.3 \times 10^{4}$ \\
\hline
\end{tabular}

\section{Acknowledgments}

The Fermi LAT Collaboration acknowledges support from a number of agencies and institutes for both development and the operation of the LAT as well as scientific data analysis. These include NASA and DOE in the United States, CEA/Irfu and IN2P3/CNRS in France, ASI and INFN in Italy, MEXT, KEK, and JAXA in Japan, and the K. A. Wallenberg Foundation, the Swedish Research Council and the National Space Board in Sweden. Additional support from INAF in Italy and CNES in France for science analysis during the operations phase is also gratefully acknowledged.

\section{References}

F. W. Stecker., 1970, Astrophys. Space Sci, 6, 377.

F. Lebrun., et al. 1983, Astrophysical Journal, 274, 231.

J. B. G M., Bloemen et al. 1984, Astronomy \& Astrophysics, $139,37$.

S. D. Hunter., et al. 1994, Astrophysical Journal, 436, 216.

S. W. Digel., et al. 1999, Astrophysical Journal, 520, 196.

W. B. Atwood., et al. 2009, Astrophysical Journal, 697, 1071.

P. M. W. Kalberla., et al. 2005, Astronomy \& Astrophysics, 440, 775 .
T. M. Dame., et al. 2001, Astrophysical Journal, 547, 792.

I. A. Grenier., et al. 2005, Science, 307, 1292.

A. A. Abdo., et al. 2010, Astrophysical Journal, 710, 133.

M. Ackermann., et al. 2012, Astrophysical Journal, 755,12 .

R. Rando., et al. 2009, arXiv:0907.0626

J. R. Mattox., et al. 1996, ApJ, 461, 396

A. A. Abdo., et al. 2010, Astrophysical Journal Supplement Series, 188, 405.

M. Ackermann., et al. 2011, Astrophysical Journal, 726,81 .

A. W. Strong., and I. V. Moskalenko., 1998, Astrophysical Journal, 509, 212.

A. A. Abdo., et al. 2009, Phys, Rev, Lett., 103, 251101

A. A. Abdo., et al. 2009, ApJ, 703, 1249.

M. Ackermann., et al. 2012, Astrophysical Journal, 750,3 .

T. Sanuki., et al. 2000, ApJ, 545, 1135.

J. Alcaraz., et al. 2000, Phys. Lett. B., 472, 215.

M. Mori., 2009, Astroparticle Physics, 31, 341.

P. Blasi., and E. Amato., 2012, J. Cosmol. Astropart. Phys, JCAP01(2012a)010.

P. Blasi., and E. Amato., 2012, J. Cosmol. Astropart. Phys, JCAP01(2012b)011.

C. W. Allen., 1973, Astrophysical Quantities (London: Athlone)

T. M. Dame., et al. 1987, ApJ, 322, 706. 